\title{
Human infection with avian influenza $A(H 7 N 9)$ virus re-emerges in China in winter 2013
}

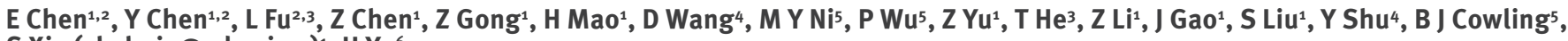

SXia (shchxia@cdc.zj.cn) ${ }^{1}, \mathrm{H} \mathrm{Yu}^{6}$

1. Department of Infectious Diseases, Zhejiang Provincial Centre for Disease Control and Prevention, Hangzhou, Zhejiang Province, China

2. These authors contributed equally to this work

3. Department of Infectious Diseases, Shaoxing Centre for Disease Control and Prevention, Shaoxing, Zhejiang Province, China

4. National Institute for Viral Disease Control and Prevention, Chinese Center for Disease Control and Prevention, Key Laboratory for Medical Virology, National Health and Family Planning Commission, Beijing, China

5. School of Public Health, Li Ka Shing Faculty of Medicine, The University of Hong Kong, Hong Kong Special Administrative Region, China

6. Division of Infectious Disease, Key Laboratory of Surveillance and Early-warning on Infectious Disease, Chinese Center for Disease Control and Prevention, Beijing, China

Citation style for this article:

Chen E, Chen Y, Fu L, Chen Z, Gong Z, Mao H, Wang D, Ni MY, Wu P, Yu Z, He T, Li Z, Gao J, Liu S, Shu Y, Cowling BJ, Xia S, Yu H. Human infection with avian influenza $\mathrm{A}\left(\mathrm{H}_{7} \mathrm{Ng}\right)$ virus re-emerges in China in winter 2013. Euro Surveill. 2013;18(43):pii=20616. Available online: http://www.eurosurveillance.org/ViewArticle.

aspx?Articleld $=20616$

Article submitted on 18 October 2013 / published on 24 October 2013

Through a national surveillance system for unexplained pneumonia, a severe case of influenza $\mathrm{A}\left(\mathrm{H}_{7} \mathrm{~N} 9\right)$ in a man in his mid-3os was identified in Zhejiang Province, China on 14 October 2013. Epidemiological and clinical findings were consistent with the patterns reported during the outbreak in spring 2013, and laboratory findings showed that the virus had $99.6 \%$ identity with earlier $\mathrm{H}_{7} \mathrm{~N} 9$ viruses identified in humans in the spring except for five mutations in the NA gene.

\section{Introduction}

Since 2004, all hospitals from the 11 cities of Zhejiang Province have participated in enhanced surveillance throughout the year of patients with pneumonia without explanation. On 14 October 2013, this system identified a new case of human infection with influenza $A\left(\mathrm{H}_{7} \mathrm{~N} 9\right)$. Here we describe how the case was discovered, and report the epidemiological, clinical and virological characteristics compared with the previous laboratory-confirmed influenza $A\left(\mathrm{H}_{7} \mathrm{~N}_{9}\right)$ cases in Zhejiang Province.

Since the first case was confirmed on 31 March 2013, a total of 135 human infections with avian influenza $\mathrm{A}\left(\mathrm{H}_{7} \mathrm{~N} 9\right)$ have been reported in China, including 45 deaths. They occurred mainly in eastern China during March and June, with the peak incidence in April [1]. After the last case with illness onset on 28 July in Guangdong Province in the south of China, no further influenza $\mathrm{A}\left(\mathrm{H}_{7} \mathrm{~N} 9\right)$ cases were reported in China until 14 October.

In Zhejiang, a province neighbouring Shanghai, the influenza season usually lasts throughout the year, with a peak between November and February. In 2013, it was one of the areas most affected by the spring outbreak of influenza $\mathrm{A}\left(\mathrm{H}_{7} \mathrm{~N} 9\right)$ during April [2]. A total of 46 confirmed cases, including 11 fatal cases, occurred in five of the 11 cities (13,000,000 inhabitants) in Zhejiang Province. Eleven of the 46 cases were clustered with four live poultry markets [2]. The decrease in the number of new human cases since mid-April may have resulted from containment measures taken by the Zhejiang authorities, such as closing live bird markets, or from a change in seasons, or a combination of both factors. Until 14 October, no confirmed cases had been reported in Zhejiang Province since 18 April. The public health response in the province had ended on 17 May.

\section{Methods}

\section{Identification of cases}

Suspected cases of $\mathrm{H}_{7} \mathrm{~N}_{9}$ virus infection are identified through the Chinese surveillance system for pneumonia of unexplained origin [3]. On identification of a suspected case, local centers for disease control and prevention (CDCs), including prefecture and provincial CDCs, conduct the initial field investigations and obtain respiratory specimens, which are shipped to the provincial CDC for $\mathrm{H}_{7} \mathrm{~N} 9$ laboratory testing [3].

\section{Definition of cases and contacts}

The definitions of probable, confirmed, severe case and surveillance case were based on Chinese guidance of diagnosis and treatment and Chinese guidance of the surveillance programme (second edition of 2013) for humans infected with $\mathrm{H}_{7} \mathrm{~N} 9$ avian influenza [3].

Based on the Chinese guidance for control and prevention of human infections with $\mathrm{H}_{7} \mathrm{~N}_{9}$ avian influenza (second edition of 2013), contacts were defined as (i) those who did not wear personal protective equipment 


\begin{tabular}{|c|c|c|c|c|c|c|c|}
\hline Segment ID & Segment & Country & $\begin{array}{l}\text { Collection } \\
\text { date }\end{array}$ & Isolate name & Originating laboratory & Submitting laboratory & Authors \\
\hline $\begin{array}{l}\mathrm{EPl} 457805 \\
\mathrm{EPI} 457804 \\
\mathrm{EPI} 457803\end{array}$ & $\begin{array}{l}\mathrm{HA}, \mathrm{NA}, \\
\mathrm{PB} 1\end{array}$ & China & $\begin{array}{l}2013-04- \\
03\end{array}$ & $\begin{array}{c}\text { A/chicken/Shanghai/ } \\
\text { S1079/2013 }\end{array}$ & $\begin{array}{l}\text { Harbin Veterinary } \\
\text { Research Institute }\end{array}$ & $\begin{array}{l}\text { Harbin Veterinary } \\
\text { Research Institute }\end{array}$ & $\begin{array}{l}\text { Zhang Q, Shi J, } \\
\text { Deng G, et al. }\end{array}$ \\
\hline $\begin{array}{l}\text { EPl440701, } \\
\text { EPI 440700, } \\
\text { EPI440699 }\end{array}$ & $\begin{array}{l}\mathrm{HA}, \mathrm{NA}, \\
\text { PB1 }\end{array}$ & China & $\begin{array}{c}2013-04^{-} \\
02\end{array}$ & $\begin{array}{c}\text { A/pigeon/Shanghai/ } \\
\text { S1069/2013 }\end{array}$ & $\begin{array}{l}\text { Harbin Veterinary } \\
\text { Research Institute }\end{array}$ & $\begin{array}{l}\text { Harbin Veterinary } \\
\text { Research Institute }\end{array}$ & $\begin{array}{l}\text { Zhang Q, Shi J, } \\
\text { Deng G, et al. }\end{array}$ \\
\hline $\begin{array}{l}\text { EPl457797, } \\
\text { EPI457796, } \\
\text { EPI457795 }\end{array}$ & $\begin{array}{l}\mathrm{HA}, \mathrm{NA}, \\
\text { PB1 }\end{array}$ & China & $\begin{array}{l}2013-04- \\
03\end{array}$ & $\begin{array}{c}\text { A/chicken/Shanghai/ } \\
\text { S1080/2013 }\end{array}$ & $\begin{array}{l}\text { Harbin Veterinary } \\
\text { Research Institute }\end{array}$ & $\begin{array}{l}\text { Harbin Veterinary } \\
\text { Research Institute }\end{array}$ & $\begin{array}{l}\text { Zhang Q, Shi J, } \\
\text { Deng G, et al. }\end{array}$ \\
\hline $\begin{array}{l}\text { EPI457845, } \\
\text { EPl457844, } \\
\text { EPI457843 }\end{array}$ & $\begin{array}{l}\mathrm{HA}, \mathrm{NA}, \\
\mathrm{PB} 1\end{array}$ & China & $\begin{array}{l}2013-05- \\
03\end{array}$ & $\begin{array}{l}\text { A/chicken/Jiangxi/ } \\
\text { SDo01/2013 }\end{array}$ & $\begin{array}{l}\text { Harbin Veterinary } \\
\text { Research Institute }\end{array}$ & $\begin{array}{l}\text { Harbin Veterinary } \\
\text { Research Institute }\end{array}$ & $\begin{array}{l}\text { Zhang Q, Shi J, } \\
\text { Deng G, et al. }\end{array}$ \\
\hline $\begin{array}{l}\mathrm{EPI} 447618, \\
\mathrm{EPI} 447716, \\
\mathrm{EPI} 447720\end{array}$ & $\begin{array}{l}\mathrm{HA}, \mathrm{NA}, \\
\text { PB1 }\end{array}$ & China & $\begin{array}{l}2013-04^{-} \\
21\end{array}$ & A/Shandong/01/2013 & Shandong CDC & $\begin{array}{c}\text { WHO Chinese National } \\
\text { Influenza Center, } \\
\text { Virology Institute, } \\
\text { Chinese CDC }\end{array}$ & $\begin{array}{l}\text { Wang D, Gao R, } \\
\text { Yang L, et al. }\end{array}$ \\
\hline $\begin{array}{l}\mathrm{EPI} 447599 \\
\mathrm{EPI} 447893 \\
\mathrm{EPI} 447897 \\
\end{array}$ & $\begin{array}{l}\mathrm{HA}, \mathrm{NA}, \\
\mathrm{PB} 1\end{array}$ & China & $\begin{array}{l}2013-03- \\
18\end{array}$ & A/Shanghai/07/2013 & Shanghai CDC & $\begin{array}{l}\text { WHO Chinese National } \\
\text { Influenza Center, } \\
\text { Virology Institute, } \\
\text { Chinese CDC }\end{array}$ & $\begin{array}{l}\text { Wang D, Gao R, } \\
\text { Yang L, et al. }\end{array}$ \\
\hline $\begin{array}{l}\text { KC885956, } \\
\text { KC885958, } \\
\text { KC885961 }\end{array}$ & $\begin{array}{l}\mathrm{HA}, \mathrm{NA}, \\
\mathrm{PB} 1\end{array}$ & China & $\begin{array}{l}2013-04- \\
18\end{array}$ & $\begin{array}{c}\text { A/Zhejiang/ } \\
\text { DTID-ZJU01/2013 }\end{array}$ & $\begin{array}{l}\text { The First Affiliated } \\
\text { Hospital, College of } \\
\text { Medicine, Zhejiang } \\
\text { University } \\
\end{array}$ & $\begin{array}{l}\text { The First Affiliated } \\
\text { Hospital, College of } \\
\text { Medicine, Zhejiang } \\
\text { University }\end{array}$ & Chen $\mathrm{H}$, Yuen $\mathrm{K}$ \\
\hline $\begin{array}{l}\mathrm{EPI} 447604 \\
\mathrm{EPI} 447829 \\
\mathrm{EPI} 447836\end{array}$ & $\begin{array}{l}\mathrm{HA}, \mathrm{NA}, \\
\text { PB1 }\end{array}$ & China & $\begin{array}{l}2013^{-04-} \\
12\end{array}$ & A/Beijing/01-A/2013 & Beijing CDC & $\begin{array}{l}\text { WHO Chinese National } \\
\text { Influenza Center, } \\
\text { Virology Institute, } \\
\text { Chinese CDC }\end{array}$ & $\begin{array}{l}\text { Wang D, Gao R, } \\
\text { Yang L, et al. }\end{array}$ \\
\hline $\begin{array}{l}\text { EPl440685, } \\
\text { EPl440684, } \\
\text { EPI440683 }\end{array}$ & $\begin{array}{l}\mathrm{HA}, \mathrm{NA}, \\
\text { PB1 }\end{array}$ & China & $\begin{array}{l}2013-04- \\
03\end{array}$ & $\begin{array}{c}\text { A/chicken/Shanghai/ } \\
\text { S1053/2013 }\end{array}$ & $\begin{array}{l}\text { Harbin Veterinary } \\
\text { Research Institute }\end{array}$ & $\begin{array}{l}\text { Harbin Veterinary } \\
\text { Research Institute }\end{array}$ & $\begin{array}{l}\text { Zhang Q, Shi J, } \\
\text { Deng G, et al. }\end{array}$ \\
\hline $\begin{array}{l}\text { EPI440693, } \\
\text { EPI } 440692 \\
\text { EPI } 440691\end{array}$ & $\begin{array}{l}\mathrm{HA}, \mathrm{NA}, \\
\mathrm{PB} 1\end{array}$ & China & $\begin{array}{c}2013-04^{-} \\
03\end{array}$ & $\begin{array}{l}\text { A/environment/ } \\
\text { Shanghai/ } \\
\text { S1088/2013 }\end{array}$ & $\begin{array}{l}\text { Harbin Veterinary } \\
\text { Research Institute }\end{array}$ & $\begin{array}{l}\text { Harbin Veterinary } \\
\text { Research Institute }\end{array}$ & $\begin{array}{l}\text { Zhang Q, Shi J, } \\
\text { Deng G, et al. }\end{array}$ \\
\hline $\begin{array}{l}\text { EPI439502, } \\
\text { EPI } 439500, \\
\text { EPI } 439501\end{array}$ & $\begin{array}{l}\mathrm{HA}, \mathrm{NA}, \\
\text { PB1 }\end{array}$ & China & $\begin{array}{l}2013-03- \\
05\end{array}$ & A/Shanghai/2/2013 & $\begin{array}{c}\text { WHO Chinese National } \\
\text { Influenza Center, } \\
\text { Virology Institute, } \\
\text { Chinese CDC }\end{array}$ & $\begin{array}{c}\text { WHO Chinese National } \\
\text { Influenza Center, } \\
\text { Virology Institute, } \\
\text { Chinese CDC }\end{array}$ & $\begin{array}{l}\text { Wang D, Gao R, } \\
\text { Yang L, et al. }\end{array}$ \\
\hline $\begin{array}{l}\text { EPI457749, } \\
\text { EPI457748, } \\
\text { EPI457747 }\end{array}$ & $\begin{array}{l}\mathrm{HA}, \mathrm{NA}, \\
\mathrm{PB} 1\end{array}$ & China & $2013-04-11$ & $\begin{array}{c}\text { A/chicken/Zhejiang/ } \\
\text { SD033/2013 }\end{array}$ & $\begin{array}{l}\text { Harbin Veterinary } \\
\text { Research Institute }\end{array}$ & $\begin{array}{l}\text { Harbin Veterinary } \\
\text { Research Institute }\end{array}$ & $\begin{array}{l}\text { Zhang Q, Shi J, } \\
\text { Deng G, et al. }\end{array}$ \\
\hline $\begin{array}{l}\text { EPI477402, } \\
\text { EPI477404, } \\
\text { EPI477400 }\end{array}$ & $\begin{array}{l}\mathrm{HA}, \mathrm{NA}, \\
\text { PB1 }\end{array}$ & China & $\begin{array}{c}2013-04^{-} \\
24\end{array}$ & A/Zhejiang/20/2013 & $\begin{array}{l}\text { Zhejiang Provincial } \\
\text { Center for Disease } \\
\text { Control and Prevention }\end{array}$ & $\begin{array}{l}\text { Zhejiang Provincial } \\
\text { Center for Disease } \\
\text { Control and Prevention }\end{array}$ & Sun Y, Zhang Y. \\
\hline $\begin{array}{l}\text { EPI } 439507, \\
\text { EPI } 439509 \\
\text { EPI } 439508\end{array}$ & $\begin{array}{l}\mathrm{HA}, \mathrm{NA}, \\
\text { PB1 }\end{array}$ & China & $\begin{array}{l}2013-03- \\
20\end{array}$ & A/Anhui/1/2013 & $\begin{array}{c}\text { WHO Chinese National } \\
\text { Influenza Center, } \\
\text { Virology Institute, } \\
\text { Chinese CDC }\end{array}$ & $\begin{array}{c}\text { WHO Chinese National } \\
\text { Influenza Center, } \\
\text { Virology Institute, } \\
\text { Chinese CDC }\end{array}$ & $\begin{array}{l}\text { Wang D, Gao R, } \\
\text { Yang L, et al. }\end{array}$ \\
\hline $\begin{array}{l}\text { EPI } 447609 \\
\text { EPI } 447780, \\
\text { EPI } 447784 \\
\end{array}$ & $\begin{array}{l}\mathrm{HA}, \mathrm{NA}, \\
\mathrm{PB} 1\end{array}$ & China & $\begin{array}{l}2013-04- \\
10\end{array}$ & A/shanghai/13/2013 & Shanghai CDC & $\begin{array}{c}\text { WHO Chinese National } \\
\text { Influenza Center, } \\
\text { Virology Institute, } \\
\text { Chinese CDC }\end{array}$ & $\begin{array}{l}\text { Wang D, Gao R, } \\
\text { Yang L, et al }\end{array}$ \\
\hline $\begin{array}{l}\text { EPI } 443028 \\
\text { EPI } 443029 \\
\text { EPI } 447942 \\
\end{array}$ & $\begin{array}{l}\mathrm{HA}, \mathrm{NA}, \\
\text { PB1 }\end{array}$ & China & $\begin{array}{l}2013-03- \\
25\end{array}$ & A/Zhejiang/01/2013 & $\begin{array}{c}\text { WHO Chinese National } \\
\text { Influenza Center, } \\
\text { Virology Institute, } \\
\text { Chinese CDC }\end{array}$ & $\begin{array}{c}\text { WHO Chinese National } \\
\text { Influenza Center, } \\
\text { Virology Institute, } \\
\text { Chinese CDC }\end{array}$ & $\begin{array}{l}\text { Wang D, Gao R, } \\
\text { Yang L, et al }\end{array}$ \\
\hline $\begin{array}{l}\text { EPI } 447614 \\
\text { EPI } 447744 \\
\text { EPI } 447748\end{array}$ & $\begin{array}{l}\mathrm{HA}, \mathrm{NA}, \\
\text { PB1 }\end{array}$ & China & $\begin{array}{l}\text { 2013-04- } \\
03\end{array}$ & A/Zhejiang/02/2013 & Zhejiang CDC & $\begin{array}{c}\text { WHO Chinese National } \\
\text { Influenza Center, } \\
\text { Virology Institute, } \\
\text { Chinese CDC }\end{array}$ & $\begin{array}{c}\text { Wang D, Gao R, } \\
\text { Yang L, et al }\end{array}$ \\
\hline $\begin{array}{l}\text { EPI457733, } \\
\text { EPI457732, } \\
\text { EPI457731 }\end{array}$ & $\begin{array}{l}\mathrm{HA}, \mathrm{NA}, \\
\mathrm{PB} 1\end{array}$ & China & $\begin{array}{c}2013-04^{-} \\
16\end{array}$ & $\begin{array}{c}\text { A/duck/Zhejiang/ } \\
\text { SC410/2013 }\end{array}$ & $\begin{array}{l}\text { Harbin Veterinary } \\
\text { Research Institute }\end{array}$ & $\begin{array}{l}\text { Harbin Veterinary } \\
\text { Research Institute }\end{array}$ & $\begin{array}{l}\text { Zhang Q, Shi J, } \\
\text { Deng G, et al. }\end{array}$ \\
\hline $\begin{array}{l}\text { EPl } 457757 \\
\text { EPI } 457756, \\
\text { EPI457755 }\end{array}$ & $\begin{array}{l}\mathrm{HA}, \mathrm{NA}, \\
\mathrm{PB} 1\end{array}$ & China & 2013-04-11 & $\begin{array}{l}\text { A/chicken/Zhejiang/ } \\
\text { SD019/2013 }\end{array}$ & $\begin{array}{l}\text { Harbin Veterinary } \\
\text { Research Institute }\end{array}$ & $\begin{array}{l}\text { Harbin Veterinary } \\
\text { Research Institute }\end{array}$ & $\begin{array}{l}\text { Zhang Q, Shi J, } \\
\text { Deng G, et al. }\end{array}$ \\
\hline $\begin{array}{l}\text { EPl477410, } \\
\text { EPI 477412, } \\
\text { EPI477408 }\end{array}$ & $\begin{array}{l}\mathrm{HA}, \mathrm{NA}, \\
\mathrm{PB} 1\end{array}$ & China & $\begin{array}{c}2013-10- \\
14\end{array}$ & A/Zhejiang/22/2013 & $\begin{array}{l}\text { Zhejiang Provincial } \\
\text { Center for Disease } \\
\text { Control and Prevention }\end{array}$ & $\begin{array}{l}\text { Zhejiang Provincial } \\
\text { Center for Disease } \\
\text { Control and Prevention }\end{array}$ & Sun Y, Zhang Y. \\
\hline
\end{tabular}




\begin{tabular}{|c|c|c|c|c|c|c|c|}
\hline Segment ID & Segment & Country & $\begin{array}{l}\text { Collection } \\
\text { date }\end{array}$ & Isolate name & $\begin{array}{l}\text { Originating } \\
\text { laboratory }\end{array}$ & $\begin{array}{l}\text { Submitting } \\
\text { laboratory }\end{array}$ & Authors \\
\hline $\begin{array}{l}\text { EPI 457861, } \\
\text { EPl457860, } \\
\text { EPI457859 }\end{array}$ & $\begin{array}{l}\mathrm{HA}, \mathrm{NA}, \\
\text { PB1 }\end{array}$ & China & $\begin{array}{l}2013-04^{-} \\
16\end{array}$ & $\begin{array}{l}\text { A/chicken/Jiangsu/ } \\
\text { SCo99/2013 }\end{array}$ & $\begin{array}{l}\text { Harbin Veterinary } \\
\text { Research Institute }\end{array}$ & $\begin{array}{l}\text { Harbin Veterinary } \\
\text { Research Institute }\end{array}$ & $\begin{array}{l}\text { Zhang Q, Shi J, } \\
\text { Deng G, et al. }\end{array}$ \\
\hline $\begin{array}{l}\text { EPl } 457789 \\
\text { EPI } 457788, \\
\text { EPI } 457787\end{array}$ & $\begin{array}{l}\text { HA, NA, } \\
\text { PB1 }\end{array}$ & China & $\begin{array}{c}2013-04- \\
03\end{array}$ & $\begin{array}{c}\text { A/chicken/Shanghai/ } \\
\text { S1358/2013 }\end{array}$ & $\begin{array}{l}\text { Harbin Veterinary } \\
\text { Research Institute }\end{array}$ & $\begin{array}{l}\text { Harbin Veterinary } \\
\text { Research Institute }\end{array}$ & $\begin{array}{l}\text { Zhang Q, Shi J, } \\
\text { Deng G, et al. }\end{array}$ \\
\hline $\begin{array}{l}\text { KFo55468, } \\
\text { KFo55470, } \\
\text { KFo55467 }\end{array}$ & $\begin{array}{l}\mathrm{HA}, \mathrm{NA}, \\
\text { PB1 }\end{array}$ & China & 2013-04 & A/Zhejiang/HZ1/2013 & $\begin{array}{c}\text { First Affiliated } \\
\text { Hospital, School of } \\
\text { Medicine, Zhejiang } \\
\text { University }\end{array}$ & $\begin{array}{c}\text { First Affiliated } \\
\text { Hospital, School of } \\
\text { Medicine, Zhejiang } \\
\text { University }\end{array}$ & $\begin{array}{l}\text { Wu H, Wu N, } \\
\text { Guo C, et al. }\end{array}$ \\
\hline $\begin{array}{l}\text { EPl } 457627, \\
\text { EPI } 457629, \\
\text { EPl } 457628\end{array}$ & $\begin{array}{l}\text { HA, NA, } \\
\text { PB1 }\end{array}$ & China & $\begin{array}{c}2013-04- \\
03\end{array}$ & $\begin{array}{l}\text { A/pigeon/Shanghai/ } \\
\text { S1423/2013 }\end{array}$ & $\begin{array}{l}\text { Harbin Veterinary } \\
\text { Research Institute }\end{array}$ & $\begin{array}{l}\text { Harbin Veterinary } \\
\text { Research Institute }\end{array}$ & $\begin{array}{l}\text { Zhang Q, Shi J, } \\
\text { Deng G, et al. }\end{array}$ \\
\hline $\begin{array}{l}\text { EPI } 447612 \\
\text { EPI } 447758 \\
\text { EPI } 447762\end{array}$ & $\begin{array}{l}\mathrm{HA}, \mathrm{NA}, \\
\mathrm{PB} 1\end{array}$ & China & $\begin{array}{l}2013-04^{-} \\
07\end{array}$ & A/Shanghai/8/2013 & Shanghai CDC & $\begin{array}{c}\text { WHO Chinese } \\
\text { National Influenza } \\
\text { Center, Virology } \\
\text { Institute, Chinese } \\
\text { CDC }\end{array}$ & $\begin{array}{l}\text { Wang D, Gao R, } \\
\text { Yang L, et al. }\end{array}$ \\
\hline $\begin{array}{l}\text { EPI457659, } \\
\text { EPl457661, } \\
\text { EPI457660 }\end{array}$ & $\begin{array}{l}\mathrm{HA}, \mathrm{NA}, \\
\text { PB1 }\end{array}$ & China & $\begin{array}{l}2013-04^{-} \\
03\end{array}$ & $\begin{array}{l}\text { A/environment/Shanghai/ } \\
\text { S1438/2013 }\end{array}$ & $\begin{array}{l}\text { Harbin Veterinary } \\
\text { Research Institute }\end{array}$ & $\begin{array}{l}\text { Harbin Veterinary } \\
\text { Research Institute }\end{array}$ & $\begin{array}{l}\text { Zhang Q, Shi J, } \\
\text { Deng G, et al. }\end{array}$ \\
\hline $\begin{array}{l}\text { EPI457675, } \\
\text { EPI457677, } \\
\text { EPI457676 }\end{array}$ & $\begin{array}{l}\mathrm{HA}, \mathrm{NA}, \\
\mathrm{PB} 1\end{array}$ & China & $\begin{array}{c}2013-04- \\
03\end{array}$ & $\begin{array}{c}\text { A/environment/Shanghai/ } \\
\text { S1436/2013 }\end{array}$ & $\begin{array}{l}\text { Harbin Veterinary } \\
\text { Research Institute }\end{array}$ & $\begin{array}{l}\text { Harbin Veterinary } \\
\text { Research Institute }\end{array}$ & $\begin{array}{l}\text { Zhang Q, Shi J, } \\
\text { Deng G, et al. }\end{array}$ \\
\hline $\begin{array}{l}\text { EPl } 457667, \\
\text { EPl } 457669, \\
\text { EPl } 457668\end{array}$ & $\begin{array}{l}\mathrm{HA}, \mathrm{NA}, \\
\text { PB1 }\end{array}$ & China & $\begin{array}{l}2013-04^{-} \\
03\end{array}$ & $\begin{array}{l}\text { A/environment/Shanghai/ } \\
\text { S1437/2013 }\end{array}$ & $\begin{array}{l}\text { Harbin Veterinary } \\
\text { Research Institute }\end{array}$ & $\begin{array}{l}\text { Harbin Veterinary } \\
\text { Research Institute }\end{array}$ & $\begin{array}{l}\text { Zhang Q, Shi J, } \\
\text { Deng G, et al. }\end{array}$ \\
\hline $\begin{array}{l}\text { EPI 457651, } \\
\text { EPI457653, } \\
\text { EPI457652 }\end{array}$ & $\begin{array}{l}\mathrm{HA}, \mathrm{NA}, \\
\text { PB1 }\end{array}$ & China & $\begin{array}{l}\text { 2013-04- } \\
03\end{array}$ & $\begin{array}{l}\text { A/environment/Shanghai/ } \\
\text { S1439/2013 }\end{array}$ & $\begin{array}{l}\text { Harbin Veterinary } \\
\text { Research Institute }\end{array}$ & $\begin{array}{l}\text { Harbin Veterinary } \\
\text { Research Institute }\end{array}$ & $\begin{array}{l}\text { Zhang Q, Shi J, } \\
\text { Deng G, et al. }\end{array}$ \\
\hline $\begin{array}{l}\text { EPI 447596, } \\
\text { EPI } 447916, \\
\text { EPI } 447920\end{array}$ & $\begin{array}{l}\mathrm{HA}, \mathrm{NA} \\
\text { PB1 }\end{array}$ & China & $\begin{array}{l}\text { 2013-03- } \\
30\end{array}$ & A/Jiangsu/01/2013 & Jiangsu CDC & $\begin{array}{c}\text { WHO Chinese } \\
\text { National Influenza } \\
\text { Center, Virology } \\
\text { Institute, Chinese } \\
\text { CDC } \\
\end{array}$ & $\begin{array}{l}\text { Wang D, Gao R, } \\
\text { Yang L, et al. }\end{array}$ \\
\hline $\begin{array}{l}\text { EPI } 457851 \\
\text { EPI } 457853 \\
\text { EPI } 457852\end{array}$ & $\begin{array}{l}\mathrm{HA}, \mathrm{NA}, \\
\text { PB1 }\end{array}$ & China & $\begin{array}{c}2013-04- \\
16\end{array}$ & $\begin{array}{l}\text { A/chicken/Jiangsu/ } \\
\text { SC537/2013 }\end{array}$ & $\begin{array}{l}\text { Harbin Veterinary } \\
\text { Research Institute }\end{array}$ & $\begin{array}{l}\text { Harbin Veterinary } \\
\text { Research Institute }\end{array}$ & $\begin{array}{l}\text { Zhang Q, Shi J, } \\
\text { Deng G, et al. }\end{array}$ \\
\hline $\begin{array}{l}\text { KC899667, } \\
\text { KC899669, } \\
\text { KC899671 }\end{array}$ & $\begin{array}{l}\mathrm{HA}, \mathrm{NA}, \\
\text { PB1 }\end{array}$ & China & 2013-04 & $\begin{array}{l}\text { A/chicken/Zhejiang/ } \\
\text { DTID-ZJU01/2013 }\end{array}$ & $\begin{array}{c}\text { First Affiliated } \\
\text { Hospital, School of } \\
\text { Medicine, Zhejiang } \\
\text { University }\end{array}$ & $\begin{array}{c}\text { First Affiliated } \\
\text { Hospital, School of } \\
\text { Medicine, Zhejiang } \\
\text { University }\end{array}$ & $\begin{array}{l}\text { Wu H, Wu N, } \\
\text { Yao H, et al. }\end{array}$ \\
\hline $\begin{array}{l}\text { EPI457619, } \\
\text { EPI457621, } \\
\text { EPI457620 }\end{array}$ & $\begin{array}{l}\mathrm{HA}, \mathrm{NA}, \\
\mathrm{PB} 1\end{array}$ & China & $\begin{array}{l}2013-04- \\
17\end{array}$ & $\begin{array}{l}\text { A/wild pigeon/Jiangsu/ } \\
\text { SDo01/2013 }\end{array}$ & $\begin{array}{l}\text { Harbin Veterinary } \\
\text { Research Institute }\end{array}$ & $\begin{array}{l}\text { Harbin Veterinary } \\
\text { Research Institute }\end{array}$ & $\begin{array}{l}\text { Zhang Q, Shi J, } \\
\text { Deng G, et al. }\end{array}$ \\
\hline $\begin{array}{l}\text { EPI 457763, } \\
\text { EPI457765, } \\
\text { EPI457764 }\end{array}$ & $\begin{array}{l}\mathrm{HA}, \mathrm{NA}, \\
\text { PB1 }\end{array}$ & China & $\begin{array}{l}2013-04- \\
22\end{array}$ & $\begin{array}{l}\text { A/chicken/Zhejiang/ } \\
\text { SDo07/2013 }\end{array}$ & $\begin{array}{l}\text { Harbin Veterinary } \\
\text { Research Institute }\end{array}$ & $\begin{array}{l}\text { Harbin Veterinary } \\
\text { Research Institute }\end{array}$ & $\begin{array}{l}\text { Zhang Q, Shi J, } \\
\text { Deng G, et al. }\end{array}$ \\
\hline $\begin{array}{l}\text { EPI } 447598 \\
\text { EPl } 447903 \\
\text { EPI } 447910\end{array}$ & $\begin{array}{l}\mathrm{HA}, \mathrm{NA}, \\
\mathrm{PB} 1\end{array}$ & China & $\begin{array}{l}2013-04- \\
02\end{array}$ & A/Shanghai/05/2013 & Shanghai CDC & $\begin{array}{c}\text { WHO Chinese } \\
\text { National Influenza } \\
\text { Center, Virology } \\
\text { Institute, Chinese } \\
\text { CDC }\end{array}$ & $\begin{array}{l}\text { Wang D, Gao R, } \\
\text { Yang L, et al. }\end{array}$ \\
\hline $\begin{array}{l}\text { EPI 439489, } \\
\text { EPI } 439486, \\
\text { EPI } 439487\end{array}$ & $\begin{array}{l}\mathrm{HA}, \mathrm{NA}, \\
\text { PB1 }\end{array}$ & China & $\begin{array}{l}2013-02- \\
26\end{array}$ & A/Shanghai/1/2013 & $\begin{array}{c}\text { WHO Chinese } \\
\text { National Influenza } \\
\text { Center }\end{array}$ & $\begin{array}{c}\text { WHO Chinese } \\
\text { National Influenza } \\
\text { Center }\end{array}$ & $\begin{array}{l}\text { Wang D, Gao R, } \\
\text { Yang L, et al. }\end{array}$ \\
\hline JQ906576 & $\mathrm{HA}$ & China & 2011-06 & $\begin{array}{c}\text { A/duck/ } \\
\text { Zhejiang/12/2011(H7N3) }\end{array}$ & $\begin{array}{c}\text { Institute of } \\
\text { Bioengineering, } \\
\text { Zhejiang Academy } \\
\text { of Medical Sciences }\end{array}$ & $\begin{array}{c}\text { Institute of } \\
\text { Bioengineering, } \\
\text { Zhejiang Academy of } \\
\text { Medical Sciences }\end{array}$ & $\begin{array}{l}\text { Hai-Bo W, } \\
\text { Ru-Feng L, } \\
\text { En-Kang W, } \\
\text { et al. }\end{array}$ \\
\hline KF259698 & NA & China & 2011 & $\begin{array}{c}\text { A/duck/ } \\
\text { Jiangxi/21714/2011(H11N9) }\end{array}$ & $\begin{array}{l}\text { Centre of Influenza } \\
\text { Research, School of } \\
\text { Public Health, The } \\
\text { University of Hong } \\
\text { Kong }\end{array}$ & $\begin{array}{c}\text { Centre of Influenza } \\
\text { Research, School } \\
\text { of Public Health, } \\
\text { The University of } \\
\text { Hong Kong }\end{array}$ & $\begin{array}{l}\text { Lam T, Wang J, } \\
\text { Shen Y, et al. }\end{array}$ \\
\hline FJ581435 & PB1 & China & 2007 & $\begin{array}{l}\text { A/chicken/Zhejiang/ } \\
\mathrm{HJ} / 2007\left(\mathrm{H}_{9} \mathrm{~N} 2\right)\end{array}$ & $\begin{array}{l}\text { Animal Husbandry } \\
\text { and Veterinary } \\
\text { Medicine of } \\
\text { Fujian Academy } \\
\text { of Agricultural } \\
\text { Sciences } \\
\end{array}$ & $\begin{array}{l}\text { Animal Husbandry } \\
\text { and Veterinary } \\
\text { Medicine of } \\
\text { Fujian Academy of } \\
\text { Agricultural Sciences }\end{array}$ & Wan C, Huang \\
\hline
\end{tabular}


Timeline of potential exposures and medical consultation, laboratory-confirmed influenza A(H7N9) case, Shaoxing city, Zhejiang Province, China, October 2013

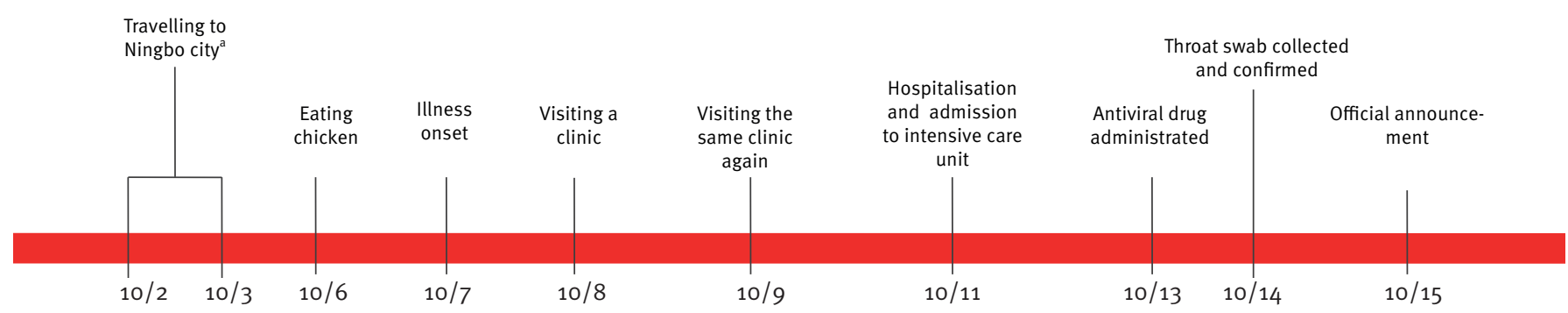

a Ningbo city is a neighbouring city to Shaoxing.

when diagnosing and treating suspected or confirmed cases or otherwise taking care of the patient; (ii) those who lived together or were in close contact with a suspected or confirmed case within 10 days of illness onset; (iii) those the epidemiologist determined as close contacts [4].

\section{Data collection}

All available medical records were reviewed by three clinicians, using a standardised data collection tool. Furthermore, epidemiologists and local public health doctors interviewed the patient's relatives, colleges and medical staff using a standard questionnaire. Pharyngeal swabs from the patient and his 25 contacts $(n=26$ in total), as well as nine environmental specimens from a live bird market nearby the patient's living place, were collected and submitted to Zhejiang CDC at $4^{\circ} \mathrm{C}$ for detection of influenza $\mathrm{A}\left(\mathrm{H}_{7} \mathrm{~N} 9\right)$ RNA.

\section{Laboratory testing}

Viral RNA was extracted using Qiagen RNeasy Mini Kit. Real-time RT-PCR was used to detect influenza type A, subtype $\mathrm{H}_{7}$ and $\mathrm{N}_{9}$ with the protocol and specific primer and probe sets provided by China CDC [5]. Seasonal influenza viruses $\left(A_{1}, A_{3}\right.$, or $\left.B\right)$, and $\mathrm{H}_{5} \mathrm{~N}_{1}$ viruses were also tested by real-time RT-PCR [5]. Complete genomic fragments of the $\mathrm{H}_{7} \mathrm{~N} 9$ virus were amplified directly from the clinical sample, and sequencing was performed with an $\mathrm{ABI} 3730 \mathrm{XL}$ automatic DNA analyser. The nucleotide sequences were determined by dideoxy sequencing, using an ABI Prism BigDye Terminator cycle sequencing kit. Phylogenetic trees were constructed by maximum likelihood method with GTR+I+Г4 model using MEGA 5.1 to estimate the relationship with selected influenza $A$ virus strains obtained from the Global Initiative on Sharing Avian Influenza Data (GISAID) database. The sequences from reference strains used in the genetic analysis were obtained from the EpiFlu database of the GISAID
(Table 1). The full genome sequence was submitted to GISAID (A/Zhejiang/22/2013(H7N9); accession no. EPI477399-414).

\section{Case description}

A male case in his mid-30s was diagnosed with laboratory-confirmed influenza $A\left(\mathrm{H}_{7} \mathrm{~N} 9\right)$ virus in Shaoxing City on 14 Oct 2013. His illness began on 7 October. The patient had no smoking history, and had no occupational exposure to poultry. He has been living in Shaoxing city for seven years, sharing with one colleague a dormitory located $1 \mathrm{~km}$ away from his daily workplace. The dormitory is located in a village where free-range chickens are kept. The patient did not report any contact with patients with influenza-like illness or visits to live-bird and animals markets within 10 days of illness onset except that he travelled to a neighbouring city on 2-3 October and ate cooked chicken on 6 October.

Illness began with influenza-like symptoms; including cough and $40.5^{\circ} \mathrm{C}$ fever on 7 October. The patient consulted a township hospital twice, on 8 and 9 October. He was first admitted to the township hospital on the morning of 11 October and then transferred to Shaoxing municipality hospital because of progressive dyspnoea and shortness of breath. At midnight on 11 October, his condition deteriorated and he was transferred to the intensive care unit. The patient was reported to Zhejiang $\mathrm{CDC}$ as a suspected influenza $\mathrm{A}\left(\mathrm{H}_{7} \mathrm{N9}\right)$ case on 14 October. One throat swab was collected on October 14 and tested positive for influenza $A\left(\mathrm{H}_{7} \mathrm{~N} 9\right)$ with realtime PCR on the same day. The government announced the case on 15 October (Figure 1).

A chest radiograph on 9 October revealed bilateral interstitial pneumonia, and consolidation was noted in a chest computed tomography scan on 11 October. The results of the clinical biomarkers are listed in 
TABLE 2

Clinical characteristics at admission, laboratory-confirmed influenza A(H7N9) case, Shaoxing City, Zhejiang Province, China, October 2013

\begin{tabular}{|c|c|c|}
\hline Characteristics & Patient & Normal value \\
\hline \multicolumn{3}{|c|}{ Clinical symptoms and signs } \\
\hline Fever & $40.5^{\circ} \mathrm{C}$ & - \\
\hline Cough & Yes & - \\
\hline $\begin{array}{l}\text { Cough with blood- } \\
\text { tinged sputum }\end{array}$ & Yes & - \\
\hline Shortness of breath & Yes & - \\
\hline Dyspnoea & Yes & - \\
\hline Chest pain & No & - \\
\hline Abdominal pain & No & - \\
\hline Diarrhoea & No & - \\
\hline Nausea & Yes & - \\
\hline Vomiting & Yes & - \\
\hline Skin ecchymosis & No & - \\
\hline Coma & Yes & - \\
\hline \multicolumn{3}{|l|}{ Blood cell count } \\
\hline White blood cell & $3.51 \times 109$ cells/L & $3.5-9.5 \times 109$ cells $/ L$ \\
\hline Neutrophils & $3.34 \times 109$ cells $/ L$ & $\begin{array}{c}1.8-6.3 \times 109 \\
\text { cells/L }\end{array}$ \\
\hline Lymphocytes & $0.12 \times 109$ cells $/ \mathrm{L}$ & $1.1-3.2 \times 109$ cells/L \\
\hline Platelets & $172 \times 109$ cells/L & $\begin{array}{c}125-320 \times 109 \\
\text { cells/L }\end{array}$ \\
\hline \multicolumn{3}{|l|}{ Biomarker } \\
\hline $\begin{array}{l}\text { Alanine } \\
\text { aminotransferase }\end{array}$ & $75 \mathrm{U} / \mathrm{L}$ & $9.0-50 \mathrm{U} / \mathrm{L}$ \\
\hline $\begin{array}{l}\text { Aspartate } \\
\text { aminotransferase }\end{array}$ & $165.2 \mathrm{U} / \mathrm{L}$ & $15-40 \mathrm{U} / \mathrm{L}$ \\
\hline $\begin{array}{l}\text { Lactate } \\
\text { dehydrogenase }\end{array}$ & $1,050.1 \mathrm{U} / \mathrm{L}$ & $109.0-245 \mathrm{U} / \mathrm{L}$ \\
\hline Creatine kinase & $16,737 \mathrm{U} / \mathrm{L}$ & $38-174 \mathrm{U} / \mathrm{L}$ \\
\hline C-reactive protein & $184.07 \mathrm{mg} / \mathrm{L}$ & $\mathrm{o}-8 \mathrm{mg} / \mathrm{L}$ \\
\hline Prothrombin time & $12.8 \mathrm{~s}$ & $11-15 \mathrm{~s}$ \\
\hline \multicolumn{3}{|l|}{ Blood gas analysis } \\
\hline Oxygen tension & $56.3 \mathrm{mmHg}$ & $83-108 \mathrm{mmHg}$ \\
\hline $\begin{array}{l}\text { Carbon dioxide } \\
\text { tension }\end{array}$ & $22.0 \mathrm{mmHg}$ & $22.0-29.0 \mathrm{mmHg}$ \\
\hline $\begin{array}{l}\text { Blood oxygen } \\
\text { saturation }\end{array}$ & $89.2 \%$ & $95-98 \%$ \\
\hline
\end{tabular}

Table 2. The case was diagnosed as a laboratoryconfirmed case of influenza $\mathrm{A}(\mathrm{H} 7 \mathrm{~N}$ 9) infection with severe pneumonia combined with two complications: acute respiratory distress syndrome (ARDS) and acute respiratory failure (type I). Treatment with oseltamivir was started at a dose of $150 \mathrm{mg}$ twice daily on 13 October. Endotracheal intubation and mechanical ventilator support (positive end expiratory pressure, PEEP) were given on 11 October because of acute respiratory failure. His condition continued to worsen despite further treatments including oxygen, antibiotic therapy (cefuroxime sodium + levofloxacin lactate + imipenem/

\begin{tabular}{|c|c|c|}
\hline Characteristics & Patient & Normal value \\
\hline \multicolumn{3}{|l|}{ Chest findings } \\
\hline $\begin{array}{l}\text { Chest X-ray on } 8 \\
\text { October }\end{array}$ & $\begin{array}{l}\text { Bilateral lung } \\
\text { markings increased }\end{array}$ & - \\
\hline $\begin{array}{l}\text { Chest computed } \\
\text { tomography on } 11 \\
\text { October }\end{array}$ & $\begin{array}{l}\text { Bilateral interstitial } \\
\text { pneumonia and } \\
\text { consolidation }\end{array}$ & - \\
\hline \multicolumn{3}{|l|}{ Complications } \\
\hline Septic shock & No & - \\
\hline Respiratory failure & Yes & - \\
\hline $\begin{array}{l}\text { Acute respiratory } \\
\text { distress syndrome }\end{array}$ & Yes & - \\
\hline Acute renal damage & No & - \\
\hline Encephalopathy & No & - \\
\hline $\begin{array}{l}\text { Multiple organ } \\
\text { failure }\end{array}$ & No & - \\
\hline $\begin{array}{l}\text { Diffuse } \\
\text { intravascular } \\
\text { coagulation }\end{array}$ & No & - \\
\hline $\begin{array}{l}\text { Secondary } \\
\text { infections }\end{array}$ & No & - \\
\hline \multicolumn{3}{|l|}{ Treatment } \\
\hline Oxygen therapy & Yes & - \\
\hline $\begin{array}{l}\text { Extracorporeal } \\
\text { membrane } \\
\text { oxygenation }\end{array}$ & No & - \\
\hline $\begin{array}{l}\text { Continuous renal } \\
\text { replacement therapy }\end{array}$ & No & - \\
\hline Antibiotic therapy & $\begin{array}{c}\text { Cefuroxime sodium } \\
+ \text { levofloxacin } \\
\text { lactate + imipenem/ } \\
\text { cilastatin }\end{array}$ & - \\
\hline Antiviral agent & Yes & - \\
\hline $\begin{array}{l}\text { Glucocorticoid } \\
\text { therapy }\end{array}$ & No & - \\
\hline $\begin{array}{l}\text { Intravenous } \\
\text { immunoglobulin } \\
\text { therapy }\end{array}$ & Yes & - \\
\hline $\begin{array}{l}\text { Mechanical } \\
\text { ventilation }\end{array}$ & $\begin{array}{c}\text { Positive end } \\
\text { expiratory pressure }\end{array}$ & - \\
\hline
\end{tabular}

cilastatin) and intravenous immunoglobulin therapy (Table 2). As of 24 October, the patient has remained in intensive care and his condition remains serious.

The case had 25 close contacts, including 21 healthcare workers, one sibling, one workplace colleague, one patient exposed in the same ward and one roommate in the dormitory. All contacts without clinical symptoms were negative for influenza $A\left(\mathrm{H}_{7} \mathrm{~N}\right.$ 9) virus RNA by real-time RT-PCR performed on 15 October during the first five days of medical observation. 


\section{FIGURE 2}

Distribution of the human influenza A (H7N9) case and the live poultry and wholesale market in Shaoxing city, Zhejiang Province, China, October 2013

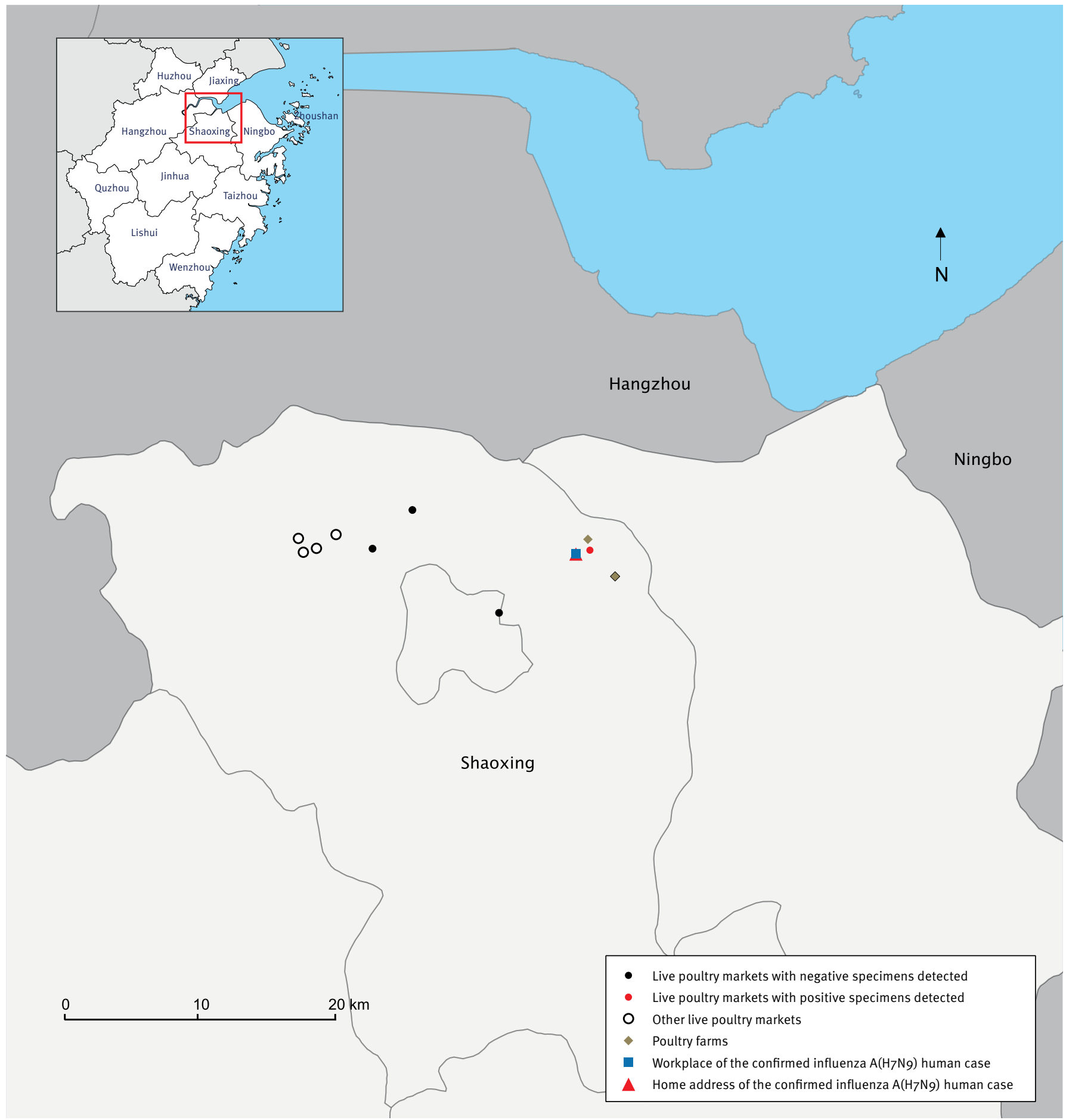

\section{Environmental samples}

Two of the nine environmental samples collected from the live poultry market and the secondary wholesale market were positive for influenza $\mathrm{A}, \mathrm{H}_{7}$ and $\mathrm{N} 9$; the other seven environmental samples remained negative for these three targets (Figure 2).
Sequence analysis

Phylogenetic analysis showed the $\mathrm{A} /$ Zhejiang/22/2013(H7N9) (ZJ/22) virus was in the same cluster with the World Health Organization (WHO)-recommended $\mathrm{A}\left(\mathrm{H}_{7} \mathrm{~N}_{9}\right)$ vaccine virus, $\mathrm{A} /$ Anhui/1/2013(H7N9) (Figure 3). 
Phylogenetic analysis of HA, NA and PB1 genes of the reemerged influenza A (H7N9) isolate, Shaoxing City, Zhejiang Province, China, October 2013
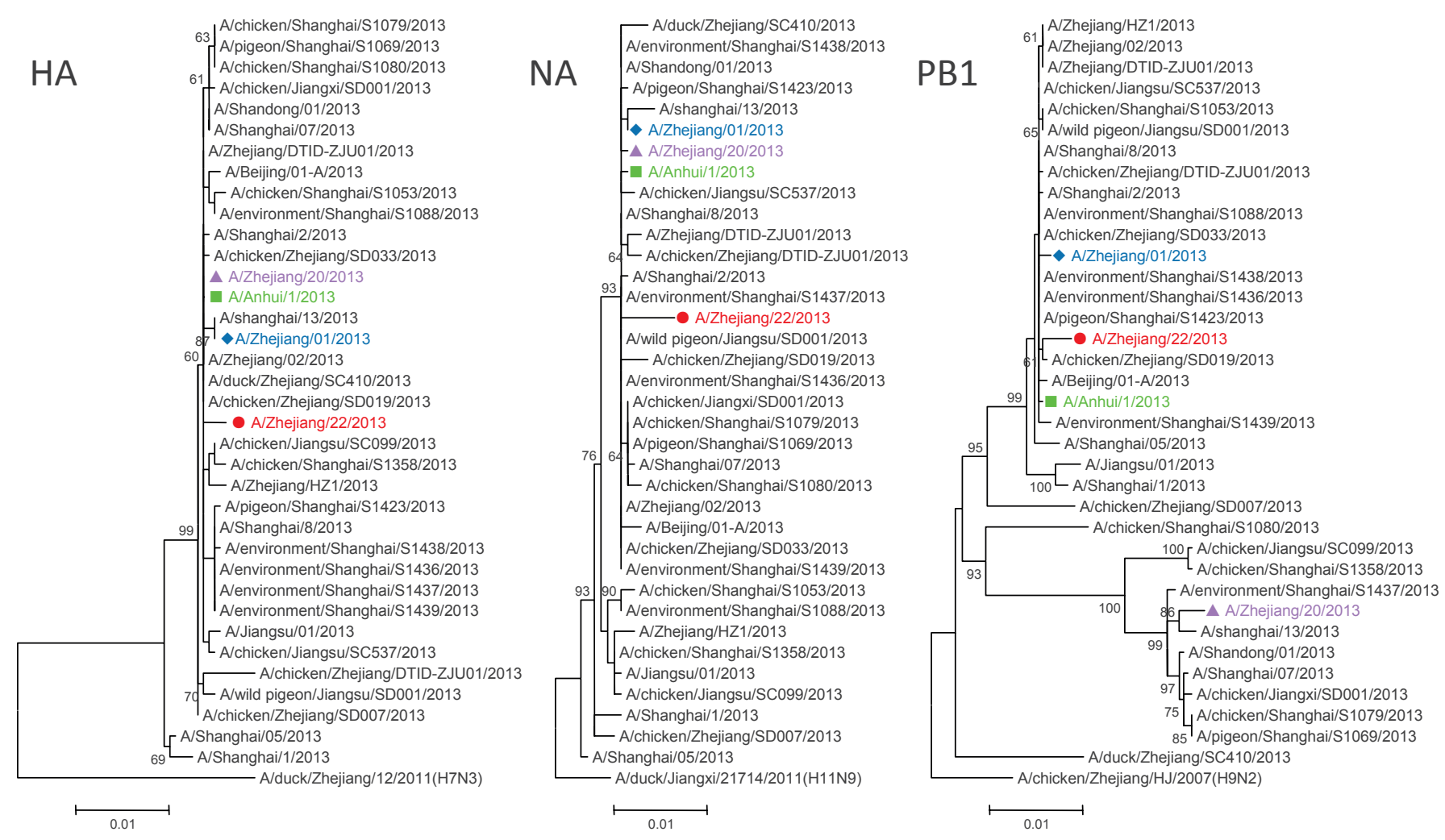

Red circle: A/Zhejiang/22/2013(H7N9), current case in October 2013; green square: $\mathrm{A} / \mathrm{Anh}$ ui $/ 1 / 2013(\mathrm{H} 7 \mathrm{~N} 9), 2013$ selected influenza vaccine strain; blue diamond: A/Zhejiang/01/2013(H7N9), the first case in the spring 2013 from Zhejiang; violet triangle: $A / Z$ hejiang/20/2013( $\mathrm{H}_{7} \mathrm{~N}$ ) $)$, the first case in the spring from Shaoxing city of Zhejiang.

The selected characteristic amino acids of the $\mathrm{ZJ} / 22$ virus were similar to other $\mathrm{H}_{7} \mathrm{~N} 9$ viruses (Table 3), which included the mammalian-adaptive mutations previously reported [5], such as Q226L in the HA gene. The $\mathrm{R} 294 \mathrm{~K}$ mutation in the $\mathrm{NA}$ gene of $\mathrm{H} 7 \mathrm{~N} 9$ virus has been reported to confer reduced sensitivity to oseltamivir and to be related to the administration of antivirals [5]. Our sample was collected only a few hours after the patient received the oseltamivir treatment, it remained $R$ in position 294 of the NA gene.* Interestingly, five amino acid mutations, V241l, K266E, N327T, N346D and $\mathrm{K} 465 \mathrm{R}$ were detected in the NA gene that had not been identified in previous $\mathrm{H}_{7} \mathrm{~N} 9$ viruses. The $\mathrm{ZJ} / 22$ viruses possessed $627 \mathrm{E}$ in the $\mathrm{PB} 2$ gene, which has been found in few of the human $\mathrm{H}_{7} \mathrm{~N} 9$ isolates and all of the $\mathrm{H}_{7} \mathrm{~N} 9$ isolates from avian or environmental samples [6]. The D701N mutation in PB2 was not detected. However, the $\mathrm{PB} 1$ gene of $\mathrm{ZJ} / 22$ presented in a different cluster with the $\mathrm{ZJ} / 20$ virus that had previously been isolated from the confirmed case in Shaoxing city in April 2013.

\section{Discussion}

We report here a severe case of laboratory-confirmed influenza $\mathrm{A}\left(\mathrm{H}_{7} \mathrm{~N}\right.$ 9) virus infection this winter in Zhejiang Province, identified through enhanced surveillance of pneumonia of unknown cause. The event shows that influenza $A\left(\mathrm{H}_{7} \mathrm{~N} 9\right)$ has returned three months after the last case had been confirmed in China in summer, and around five months since the spring outbreak in eastern China. It indicates a possible risk of a larger outbreak of influenza $A\left(\mathrm{H}_{7} \mathrm{Ng}\right)$ this winter [7]. The epidemiological, clinical and laboratory findings in this case were similar to the previous cases in Zhejiang Province this spring, with no evidence of sustained person-to-person transmission. However, this case differed from the previous cases in that it was a younger severe case with no obvious underlying diseases and no obvious recent direct contact with live poultry. Most laboratory-confirmed cases in the past had been older patients over the age of 60 years reporting recent exposure to poultry, generally at live bird markets $[3,5,7]$. Epidemiological and clinical data have been shared with WHO and other international partners. 
Selected characteristic amino acids of the A/Zhejiang/22/2013(H7N9) virus isolated from a laboratory-confirmed influenza A(H7N9) case, Shaoxing City, Zhejiang Province, China, October 2013

\begin{tabular}{|c|c|c|c|c|c|c|}
\hline Genes & Function associated & Sites & $\begin{array}{l}\text { A/Zhejiang/ } \\
22 / 2013\end{array}$ & $\begin{array}{l}\text { A/Anhui/ } \\
1 / 2013\end{array}$ & $\begin{array}{l}\text { A/Zhejiang/ } \\
1 / 2013\end{array}$ & $\begin{array}{l}\text { A/Zhejiang/ } \\
20 / 2013\end{array}$ \\
\hline \multirow{5}{*}{$\begin{array}{l}\mathrm{HA} \\
\text { ( } \mathrm{H}_{3}- \\
\text { Numbering) }\end{array}$} & $\begin{array}{l}\text { Without } \mathrm{N} \text {-glycosylation and increased } \\
\text { virus binding to human-type receptors }\end{array}$ & 160 & A & $A$ & A & A \\
\hline & Specific mutation $^{a}$ & 222 & $\mathbf{P}$ & Q & Q & Q \\
\hline & \multirow{3}{*}{ Receptor binding site } & 186 & $\mathbf{v}$ & $\mathrm{V}$ & $\mathrm{V}$ & $\mathrm{V}$ \\
\hline & & 226 & $\mathbf{L}$ & L & I & $\mathrm{L}$ \\
\hline & & 228 & G & G & G & G \\
\hline \multirow{16}{*}{ NA } & \multirow{11}{*}{ Drug resistance } & 119 & $\mathbf{R}$ & $R$ & $R$ & $R$ \\
\hline & & 120 & $\mathbf{E}$ & $\mathrm{E}$ & $\mathrm{E}$ & $\mathrm{E}$ \\
\hline & & 152 & D & D & D & $\mathrm{D}$ \\
\hline & & 153 & $\mathbf{R}$ & $R$ & $R$ & $R$ \\
\hline & & 224 & $\mathbf{I}$ & 1 & 1 & 1 \\
\hline & & 226 & $\mathbf{R}$ & $R$ & $R$ & $R$ \\
\hline & & 276 & H & $\mathrm{H}$ & $\mathrm{H}$ & $\mathrm{H}$ \\
\hline & & 278 & $\mathbf{E}$ & $\mathrm{E}$ & $\mathrm{E}$ & $\mathrm{E}$ \\
\hline & & 294 & $\mathbf{R}$ & $R$ & $R$ & $R$ \\
\hline & & 296 & $\mathbf{N}$ & $\mathrm{N}$ & $\mathrm{N}$ & $\mathrm{N}$ \\
\hline & & 372 & $\mathbf{R}$ & $R$ & $R$ & $R$ \\
\hline & \multirow{5}{*}{ Specific mutation ${ }^{a}$} & 241 & $\mathbf{I}$ & $\mathrm{V}$ & $\mathrm{V}$ & $\mathrm{V}$ \\
\hline & & 266 & $E$ & K & $\mathrm{K}$ & K \\
\hline & & 327 & $T$ & $\mathrm{~N}$ & $\mathrm{~N}$ & $\mathrm{~N}$ \\
\hline & & 346 & D & $\mathrm{N}$ & $N$ & $\mathrm{~N}$ \\
\hline & & 465 & $\mathbf{R}$ & $\mathrm{K}$ & K & K \\
\hline \multirow{2}{*}{ PB2 } & \multirow{2}{*}{ Enhanced transmission } & 627 & $\mathbf{E}$ & K & K & $E$ \\
\hline & & 701 & D & $\mathrm{D}$ & D & $\mathrm{D}$ \\
\hline $\mathrm{PB} 1-\mathrm{F}_{2}$ & Increased pathogenicity & & 90 aa & 90 aа & 90 aа & 25 aа \\
\hline $\mathrm{M} 2$ & Amantidine resistance & 31 & $\mathbf{N}$ & $\mathrm{N}$ & $\mathrm{N}$ & $\mathrm{N}$ \\
\hline
\end{tabular}

a These mutations have not been found in previously reported $\mathrm{H}_{7} \mathrm{~N}_{9}$ viruses.

The surveillance system for unexplained pneumonia covering in all hospitals of Zhejiang Province was set up since 2004 ran routinely for 10 years. In 2013, following the confirmation of the first influenza $A\left(\mathrm{H}_{7} \mathrm{~N} 9\right)$ case on 1 April, the surveillance system was expanded and strengthened, and one confirmed influenza $\mathrm{A}\left(\mathrm{H}_{7} \mathrm{~N} 9\right)$ case was discovered through this system during the spring outbreak, but no further cases were discovered until 14 October. This observation is consistent with seasonality of influenza $A\left(\mathrm{H}_{7} \mathrm{~N} 9\right)$ cases, much like human cases of influenza $\mathrm{A}\left(\mathrm{H}_{5} \mathrm{~N}_{1}\right)$ in China that have mainly been occurring in the winter months [8].

The clinical features in this new case were similar to the cases reported in the spring in Zhejiang Province and other areas of China [9]. They shared the acute onset and rapid development to severe and sustained hypoxia, accompanied by respiratory failure, ARDS, etc. Although the patient received broad-spectrum antibiotics, supportive management and mechanical ventilation, his condition deteriorated. Without underlying comorbid conditions or other risk factors, the case did not receive oral oseltamivir until seven days after disease onset, which may have contributed to disease progression as early treatment with neuraminidase inhibitors can be effective $[7,9]$.

Although no other influenza $\mathrm{A}\left(\mathrm{H}_{7} \mathrm{~N} 9\right)$ outbreaks had been identified in animals in Shaoxing city before onset of the present case's illness and although he had no history of direct exposure to birds, the isolated virus had a high degree of similarity to $\mathrm{H}_{7} \mathrm{~N} 9$ viruses 
previously reported in humans, with $98.5 \%-99.6 \%$ (see Figure 3). Five mutations in the NA gene have never been reported in those strains isolated in spring 2013. The origin of these mutations is not clear, and any potential change in pathogenicity or transmissibility remains to be determined. In addition, positive PCR results were obtained in two of the nine samples from live bird markets, which is consistent with continuing circulation of this virus in poultry and epidemic spillover leading to human cases $[10,11]$. Within 10 days before his illness onset, the case had spent two days in a rural area, and wild animals cannot be excluded as a possible source, although the incubation period would then have been very long. If influenza $A\left(\mathrm{H}_{7} \mathrm{~N} 9\right)$ transmission continues in poultry, human infections may become more frequent when temperatures decrease in China this winter.

This study had some limitations: Firstly, we were unable to comprehensively investigate the exposure and travel history because the case has been unconscious in the intensive care unit since 11 October. Secondly, medical observation of contacts is still ongoing without any positive findings to date. Thirdly, the $\mathrm{H}_{7} \mathrm{~N} 9$ positive isolates from birds from the markets have not been sequenced because the viral load in the environment was too low. Finally, we have just begun a serological survey to identify subclinical infections among local residents associated with exposures in the affected live poultry markets and poultry farms.

\section{Conclusion}

Human infection with influenza $\mathrm{A}\left(\mathrm{H}_{7} \mathrm{~N} 9\right)$ virus has reemerged in winter 2013, without substantial genetic change in the virus, signalling the potential for a new epidemic wave this winter. It is important to monitor the pandemic potential of this re-emerging virus which has apparently continued to circulate in an animal reservoir during the summer. Based on experiences in the spring, enhanced and expanded surveillance in the human and animal populations help to ensure early discovery and diagnosis of suspected cases, while hygiene campaigns and closure of live poultry markets can reduce the risk of severe cases and deaths. In particular, enhanced surveillance in poultry would be helpful if it can identify the $\mathrm{H} 7 \mathrm{~N} 9$ virus and inform early control measures before human infections occur. In the longer term, reformation of the poultry farming, distribution and purchasing system may be required to reduce human risk of infection with avian influenza viruses.

Note added in proof

A second severe laboratory-confirmed influenza $A\left(\mathrm{H}_{7} \mathrm{Ng}\right)$ case was identified on 23 October in Jiaxing City, Zhejiang Province, while this manuscript was in production. The case is a man in his late 6os with no underlying disease who lived in a rural area and whose occupation included transporting and selling poultry.
Acknowledgements

We are grateful for the exemplary contributions of Professor Zhao Hui Huang, Wei Yu and Shengfang Ren from Shaoxing city Center for Disease Control and Prevention, Zhejiang Province, China, for their collecting of the samples and epidemical data. We would also like to thank coordinator $\mathrm{Dr}$ Chengmin Wang from National Research Center for Wildlife Born Diseases, Institute of Zoology, Chinese Academy of Sciences for his professional comments. The authors thank Hoi Wa Wong and Angel Li for technical assistance.

This work was supported by grants from Provincial Medical Research Fund of

Zhejiang, China (wkj2013-2-008) and Zhejiang Leading Team of Science and Technology Innovation (grant number 2011R50021).

\section{Conflict of interests}

None declared.

Ethics Statement

For all sampling activities official permits were approved by the Chinese medical ethical committee, and the National Health and Family Planning Commission (NHFPC) approved the study. We got the written consent of collecting samples by the relatives of this patient.

Authors' contributions

Conceived and designed the experiments: Enfu Chen, Yin Chen, Lijun Fu, Zhiping Chen, Benjamin J. Cowling. Epidemiological investigation: Haiyan Mao, Dayan Wang, Michael Y. Ni, Peng Wu, Zhenyu Gong, Zhao Yu. Performed the experiments of virology: Tingting He, Zhen Li, Jian Gao. Contributed reagents/materials/analysis: Yuelong Shu. Drafted the manuscript: Shelan Liu. Conceived and coordinated the study: Shichang Xia, Hongjie Yu.

* Authors' correction:

In the first column of Table $1 B$, the segment IDs in the last three rows were amended to include one segment ID per row.

In addition, the sentence, 'Our sample was collected before the patient received the oseltamivir treatment, it remained $R$ in position 294 of the NA gene' was corrected to read, 'Our sample was collected only a few hours after the patient received the oseltamivir treatment, it remained $R$ in position 294 of the NA gene'.

These corrections were made on 25 October 2013 at the request of the authors. 


\section{References}

1. Number of confirmed human cases of avian influenza $A\left(\mathrm{H}_{7} \mathrm{~N} 9\right)$ reported to WHO. Geneva: World Health Organization. [Accessed 12 Aug 2013]. Available from: http://www.who. int/influenza/human_animal_interface/influenza_h7n9/09_ ReportWebH7NoNumber.pdf

2. Liu S, Sun J, Cai J, Miao Z, Lu M, Qin S, et al. Epidemiological, clinical and viral characteristics of fatal cases of human avian influenza $\mathrm{A}\left(\mathrm{H}_{7} \mathrm{~N} 9\right)$ virus in Zhejiang Province, China. J Infect. 2013 pii: S0163-4453(13)00239-9. doi: 10.1016/j. jinf.2013.08.007. http://dx.doi.org/10.1016/j.jinf.2013.08.007

3. National Health and Family Planning Commission of China. Chinese guideline for diagnosis and treatment in human infection with $\mathrm{H}_{7} \mathrm{~N} 9$ avian flu (2nd edition of 2013). Beijing: Chinese Center for Disease Control and Prevention. [Accessed Jun 2013]. Chinese. Available from: http://www.chinacdc.cn/ jkzt/crb/rgrgzbxqlg_5295/rgrglgyh/201304/t20130411_79740. htm

4. Li Q, Zhou L, Zhou M, Chen Z, Li F, Wu H, et al. Preliminary Report: Epidemiology of the Avian Influenza A (H7N9) Outbreak in China. N Engl J Med. 2013 Apr 24; [Epub ahead of print]. doi: 10.1056/NEJMoa1304617. http://dx.doi.org/10.1056/NEJMoa1304617

5. Gao R, Cao B, Hu Y, Feng Z, Wang D, Hu W, et al. Human infection with a novel avian-origin influenza $\mathrm{A}(\mathrm{H} 7 \mathrm{~N} 9)$ virus. $\mathrm{N}$ Engl J Med. 2013;368(20):1888-97. http://dx.doi.org/10.1056/ NEJMoa1304459. PMid:23577628.

6. Li J, Yu X, Pu X, Xie L, Sun Y, Xiao H, et al. Environmental connections of novel avian-origin $\mathrm{H}_{7} \mathrm{~N} 9$ influenza virus infection and virus adaptation to the human. Sci China Life Sci. 2013;56(6):485-92. doi: 10.1007/s11427-013-4491-3. http://dx.doi.org/10.1007/s11427-013-4491-3

7. Overview of the emergence and characteristics of the avian influenza $\mathrm{A}\left(\mathrm{H}_{7} \mathrm{~N} 9\right)$ virus. Geneva: World Health Organization; 31 May 2013]. Available from: http://www.who.int/influenza/ human_animal_interface/influenza_h7n9/WHO_H7N9_ review_31May13.pdf

8. Cowling BJ, Jin L, Lau EH, Liao Q, Wu P, Jiang H, et al.

Comparative epidemiology of human infections with avian influenza $\mathrm{A} \mathrm{H}_{7} \mathrm{~N}_{9}$ and $\mathrm{H}_{5} \mathrm{~N}_{1}$ viruses in China: a populationbased study of laboratory-confirmed cases. Lancet. 2013;382(9887):129-37.

http://dx.doi.org/10.1016/S0140-6736(13)61171-X

9. Gao HN, Lu HZ, Cao B, Du B, Shang H, Gan JH, et al. Clinical findings in 111 cases of influenza $\mathrm{A}(\mathrm{H} 7 \mathrm{~N} 9)$ virus infection. $\mathrm{N}$ Engl J Med. 2013;368 (24):2277-85. http://dx.doi.org/10.1056/ NEJMoa1305584. PMid:23697469.

10. Qi X, Qian YH, Bao CJ, Guo XL, Cui LB, Tang FY, et al. Probable person to person transmission of novel avian influenza $A\left(\mathrm{H}_{7} \mathrm{~N} 9\right)$ virus in Eastern China, 2013: epidemiological investigation. BMJ. 2013;347:f4752. http://dx.doi.org/10.1136/ bmj.f4752. PMid:23920350.

11. Chen Y, Liang W, Yang S, Wu N, Gao H, Sheng J,et al. Human infections with the emerging avian influenza $\mathrm{A} \mathrm{H} 7 \mathrm{~N}$ 9 virus from wet market poultry: clinical analysis and characterization of viral genome. Lancet. 2013;381(9881):1916-25. http://dx.doi.org/10.1016/S0140-6736(13)60903-4 